\title{
Monotileable Amenable Groups: An Application
}

\author{
Amal Mohammed Ahmed Gaweash 1,2, Hayat Yousuf Ismail Bakur ${ }^{3,4}$, \\ Mariam Almahdi Mohammed Mulla ${ }^{4,5}$ \\ ${ }^{1}$ Department of Mathematics, University of Taif, Ta'if, Makkah, KSA \\ ${ }^{2}$ University of Aldalling, Sudan \\ ${ }^{3}$ Department of Mathematics, University of Jazan, Jazan, KSA \\ ${ }^{4}$ University of Kordofan, El-Obeid, North Kordofan, Sudan \\ ${ }^{5}$ Department of Mathematics, University of Hafr Al-Batin, Hafar Al-Batin, KSA \\ Email: amal.gaweash@gmail.com, hayatdxdy@gmail.com, marimdx2014@gmail.com
}

How to cite this paper: Gaweash, A.M.A., Bakur, H.Y.I. and Mulla, M.A.M. (2021) Monotileable Amenable Groups: An Application. Open Access Library Journal, 8: e7012.

https://doi.org/10.4236/oalib.1107012

Received: November 17, 2020

Accepted: January 26, 2021

Published: January 29, 2021

Copyright (C) 2021 by author(s) and Open Access Library Inc.

This work is licensed under the Creative

Commons Attribution International

License (CC BY 4.0).

http://creativecommons.org/licenses/by/4.0/

\begin{abstract}
We study actions of countable discrete groups which are Monotileable amenable groups in the sense that there exists a mean on $X$ which is invariant under the action of $G$. Assuming that $G$ is nonamenable, we obtain structural results for the stabilizer subgroups of amenable actions which allow us to relate the first $\ell^{2}$-Betti number of $G$ with that of the stabilizer subgroups.

\section{Subject Areas}

Mathematical Analysis, Mathematical Modern Algebra and Foundation of Mathematics

\section{Keywords}

Varieties of Groups, Abelian Groups, Multiplication, Amenable Groups, Lie Group, F $\phi$ lner Sequence
\end{abstract}

\section{Introduction}

In this paper, it has been conjectured on the basis of current knowledge that the class of Monotileable amenable groups consists precisely of all groups $G$ which do not possess a subgroup isomorphic to the free group on two generators. In this paper, we will not settle this conjecture. However, this work is motivated by a desire to give an algebraic description of the class of all amenable groups. In an attempt to determine which varieties of groups consist entirely of amenable groups, the notion of a uniformly amenable group is introduced. We derive results indicating the relationships between varieties and uniformly amenable groups. An action of a discrete group $G$ on a set $X$ is said to be amenable if there 
exists a finitely additive probability measure: $\mathcal{P}(X) \rightarrow[0,1]$, henceforth called a mean, defined on the power set of $X$, which is invariant under the action of $G$ [1] [2].

\subsection{Varieties of Groups}

We present here some of the ideas and results necessary in the sequel. Further information can be obtained by consulting [3].

Let $X\left\{x_{i}: i\right.$ is a positive integer $\}$ be an alphabet. Let $X_{m}$ be the free group of rank $n$ generated by $\left\{x_{i}: 1 \leq i \leq n\right\}$ and let $X_{\infty}$ be the free group generated by $X$. If $w \in X_{n}$, we will often write $w\left(x_{1}, \cdots, x_{n}\right)$ to denote $w$.

If $G$ is any group and al, as $a_{1}, a_{2}, \cdots, a_{n} \in G$, then by $w\left(a_{1}, a_{2}, \cdots, a_{n}\right)$ for $w \in X_{n}$. We mean $\alpha(w)$ where $a$ is the unique homomorphism from $X_{n}$ into $G$ with

$$
\alpha\left(x_{i}\right) a_{i} \text { for } 1 \leq i \leq n .
$$

A word $w\left(x_{1}, x_{2}, \cdots, x_{n}\right)$ is called an identical relation or a law for a group $G$ if and only if $w\left(a_{1}, a_{2}, \cdots, a_{n}\right)=1$ for every $a_{1}, a_{2}, \cdots, a_{n}$ in $G$. A law is called trivial if it is satisfied by all groups and this happens if and only if $w=1$.

A variety of groups is the class of all groups satisfying each law in a given set of laws. If $L \subseteq X_{\infty}$ then we denote by $V(L)$ the variety of all groups for which the words in $L$ are laws.

We mention few examples of varieties. The class of all abelian groups is obviously $V\left(x_{1}^{-1} x_{2}^{-1} x_{1} x_{2}\right)$. For any positive integer $t$ the class of all solvable groups of derived length $\leq t$ form variety, the variety $V\left(x^{m}\right)$ is called the Burnside variety of exponent $m$. The class of all groups is variety we will denote by $\mathcal{O}$. A reduced free group in variety is group with a set of generators $S$ such that any map from $S$ into group in $V$ can be extended to homomorphism.

For any cardinal number $h, V$ contains reduced free group with $h$ generators. For every natural number $n, F_{n}(V)$ will denote reduced free group with $n$ generators. A reduced free group with countably infinite set of generators will be denoted by $F_{\infty}(V)$ [1] [3].

The Cartesian product of $\left\{A_{\lambda} \mid \lambda \in \Lambda\right\}$ of groups will be denoted by $C_{\lambda} \Pi_{\lambda \in \Lambda} A_{\lambda}$ and the direct product will be denoted by $\prod_{\lambda \in \Lambda} A_{\lambda}$. The direct product of a countably infinite number of groups isomorphic to a group $G$ will be denoted by $\Pi G$.

Let $V$ be a variety of groups. A set of groups $\mathfrak{D} \subseteq V$ is said to discriminate $V$ if and only if for every finite set of words $W$ in $X_{\infty}$ which are not laws for $V$ there exists $G \in \mathfrak{D}$ and elements $g_{1}, g_{2}, \cdots, g_{n} \in G$ such that

$$
w\left(g_{1}, g_{2}, \cdots, g_{n}\right) \neq 1
$$

for any $w \in W$. ( $n$ is some integer for which $W \subseteq X_{n}$.) Put another way any finite set of nonlaws for $V$ can be simultaneously falsified.

The set of all varieties of groups is partially ordered under inclusion. It is a complete lattice where $U \wedge V=U \cap V$. The variety generated by u class of 
groups $\mathcal{C}$ is denoted by $V(\mathcal{C})$ and defined by $V(\mathcal{C}) \bigcap_{V \supseteq \mathcal{C}} V$.

Multiplication can be defined on varieties where $U V$ is the variety of all extensions of groups in $U$ by groups in $V$ [2] [3].

\subsection{Lemma}

Let $V$ be a variety of groups and let $\mathfrak{D}$ be a set of groups contained in $V$. Let $M$ be a full structure whose individuals include all elements of all groups in $\mathfrak{D}$ all elements of $X_{\infty}$ and all natural numbers. Let ${ }^{*} M$ be an enlargement of $M$. Then $\mathfrak{D}$ discriminates $V$ if and only if $F_{\infty}(V)$ is isomorphic to a subgroup of a group $D \in D^{*}$.

Proof. Suppose $\mathfrak{D}$ discriminates $V$.

If $W$ is a finite set of nonlaws for $V$ and $W \subseteq X_{n}$ then there exists $D \in \mathfrak{D}$ and $d_{1}, d_{2}, \cdots, d_{n} \in D$ such that $w\left(d_{1}, d_{2}, \cdots, d_{n}\right) \neq 1$ for any $w \in W$.

Now let $Y \subseteq X_{\infty}$ consist of all nonlaws for $V$. Let $E$ be a ${ }^{*}$-finite subset of ${ }^{*} Y$ such that $Y \subseteq E$ and let e ${ }^{*} N-N$ be chosen so that $E \subseteq X_{\infty}$.

Since every element of $Y$ is a nonlaw for $V$ so is every element of $E$. We get:

There exists $D \in D^{*}$ and $d_{1}, d_{2}, \cdots, d_{w} \in D$ such that

$$
e\left(d_{1}, d_{2}, \cdots, d_{w}\right) \neq 1
$$

for every $e \in E$. In particular the only relations on the elements $d_{i}$ are identities for $V$ and so the group they generate is free reduced on an infinite set of generators and so $F_{\infty}(V)$ is certainly isomorphic to a subgroup of $D$.

On the other hand suppose $\mathfrak{D}$ does not discriminate $V$. Then there is a finite set $W$ of nonlaws for $V$ which cannot be simultaneously falsified in any $D \in \mathfrak{D}$. Suppose $W \subseteq X_{n}$. We have:

If $D \in \mathfrak{D}$ and $d_{1}, d_{2}, \cdots, d_{n} \in D$ then there exists $w \in W$ with

$$
w\left(d_{1}, d_{2}, \cdots, d_{n}\right)=1 \text {. }
$$

So, if $D \in{ }^{*} \mathfrak{D}$ and $d_{1}, d_{2}, \cdots, d_{n} \in D$ then there exists $w \in W$ with

$$
w\left(d_{1}, d_{2}, \cdots, d_{n}\right)=1 \text {. }
$$

That is, every $n$ element in any group in ${ }^{*} \mathfrak{D}$ satisfies a relation which is not a law for $V$. So $F_{\infty}(V)$ is not isomorphic to a subgroup of $D$ for any $D \in{ }^{*} \mathfrak{D}$ and the proof is complete [3].

\section{How to Build Amenable Groups}

$A \Delta B=(A \backslash B) \cup(B \backslash A)$. Groups are assumed countable and discrete.

\subsection{Definition}

(F $\phi$ lner, 1955) A group $\Gamma$ is amenable if and only if there exists a $F \phi l n e r$ sequence, i.e. a sequence of finite sets $\left\{F_{n}\right\}_{n \geq 1}$ such that for all [4] $\gamma \in \Gamma$, $\lim _{n \rightarrow \infty} \frac{\left|F_{n} \gamma \Delta F_{n}\right|}{\left|F_{n}\right|}=0$. 


\subsection{Example}

Here are a few basic examples:

- A finite group is amenable, take $F_{n}=\Gamma$ for all $n$. Then $F_{n} \gamma=F_{n}$ and $\frac{\left|F_{n} \gamma \Delta F_{n}\right|}{\left|F_{n}\right|}=0$.

- A direct union of finite groups is amenable $\Gamma=\oplus_{n \in \mathbb{N}} G_{i}$. An element $\gamma \in \Gamma$ is a sequence $\gamma_{i} \in G_{i}$, so that for some $n_{0}$ (depending on $\gamma$ ), $\forall n \geq n_{0}$, $\gamma_{n}=e G_{i}$. Take $F_{n}=\oplus_{i=0}^{n} G_{i}$, then for any $\gamma \in \Gamma, F_{n} \gamma=F_{n}$ for $n$ large enough. So $\frac{\left|F_{n} \gamma \Delta F_{n}\right|}{\left|F_{n}\right|}=0$ for $n$ large enough.

- $\mathbb{Z}$ is amenable. Take $F_{n}=[1, n]$. Without loss of generality, one can comp-ute only $\gamma>0$. Then

$$
\frac{\left|F_{n} \gamma \Delta F_{n}\right|}{\left|F_{n}\right|}=\frac{1}{n}|[1, \gamma] \cup[n+1, n+\gamma]|=2|\gamma| / n \rightarrow 0 .
$$

When the graph is finitely generated by a set for $S$ between

$\delta_{s} F_{n}=\left\{\right.$ edges between $F_{n}$ and $\left.F_{n}^{c}:=\Gamma / F_{n}\right\}$. A first useful lemma that reduces some computation is [1] [4].

\subsection{Lemma}

Assume $\Gamma$ is finitely generated. A sequence $\left\{F_{n}\right\}$ is $F \phi l n e r$ if and only if $\lim _{n \rightarrow \infty} \frac{\left|\partial F_{n}\right|}{\left|F_{n}\right|}$.

The proof is left as an exercise. An isoperimetric profile for the group is an increasing function $\mathcal{F}: R_{>0} \rightarrow R_{>0}$ such that $\exists C>0$ so that for any finite set $F, \mathcal{F}(|F|) \leq|\partial F|$. Amenable groups are groups with sub linear $\mathcal{F}$.

In L. Saloff-Coste's lectures it was shown that growth of balls which is $\geq C^{\prime} n^{d}$ implies $\mathcal{F}(x)=x^{(d-2) / d}$ works. However, putting $d=\infty$ is a risky business: there are groups which are amenable but have growth faster than any polynomial (e.g. $B S(1,2)$ from C. Pittet's lecture). It's an easy exercise to show $\mathcal{F}(x)=x^{(d-1) / d}$ is optimal for $\mathbb{Z}^{d} \quad[2]$.

Actually one can easily show that groups with "slow" volume growth are amenable. Recall a group is of sub exponential growth if $\beta_{s}(n):=|B(n)|=\mathrm{e}^{f(n)}$ for some (increasing) function $f: \mathbb{N} \rightarrow \mathbb{R}_{>0}$ satisfying $\lim _{n \rightarrow \infty} \frac{f(n)}{n}=0$.

\subsection{Proposition}

Let $\Gamma$ be a [finitely generated] group of sub exponential growth, then $\Gamma$ is amenable.

Proof: The number of edges in $\partial B_{n}$ is at most the number of vertices in the sphere of radius $n+1$ times the maximal degree of a vertex, $|S|$. 


$$
\frac{\left|\partial B_{n}\right|}{\left|B_{n}\right|} \leq|S| \frac{\left|\partial B_{n+1}\right|-\left|B_{n}\right|}{\left|B_{n}\right|}=|S|\left(\mathrm{e}^{f(n+1)-f(n)}-1\right) .
$$

The claim is that there is some sequence of integers $\left\{n_{k}\right\}_{k \geq 0}$ so that $f\left(n_{k}+1\right)-f\left(n_{k}\right)$ tends to 0 . If this is the case, then $F_{k}=B_{n k}$ would be a $\mathrm{F} \phi$ lner sequence. So assume there are no such sequences, or, in other words that $\exists \epsilon>0$ such that $\forall n>0, f(n+1)-f(n)>\epsilon$. This implies that

$$
f(k)=f(0)+\sum_{i=1}^{k}(f(t)-f(i-1))>k \epsilon .
$$

This a contradiction with $\lim _{n \rightarrow \infty} \frac{f(n)}{n}=0$. Hence the desired subsequence exists and $\Gamma$ is amenable.

Note that it is highly non-trivial to see whether the sequence of all balls works. This turns out to be true in nilpotent groups. It is open in a group of intermediate growth.

The converse of proposition 1.4 is not true: $B S(1,2)$ is amenable but has exponential growth.

Proposition 1.4 gives many groups without too much effort. The next theorem is useful to build groups out of known amenable groups [4].

\subsection{Theorem}

("The closure properties" 3)

Let $\Gamma, N$ and $\left\{\Gamma_{i}\right\}_{i \geq 0}$ be amenable groups.

1) If $H$ is a subgroup 4 of $\Gamma$ then $H$ is amenable "Subgroup".

2) If $H$ is an extension $N$ by $\Gamma(1 \rightarrow N \rightarrow H \rightarrow \Gamma \rightarrow 1)$ is an exact sequence) then $H$ is amenable "Extension".

3) If $N \Delta \Gamma$ then $H=\Gamma / N$ is amenable "Quotient".

4) If $H$ is a direct limit of the $\Gamma_{i} \geq 0$ then $H$ is amenable "Direct limit".

It's perfectly possible to prove these properties from the current definition of amenability. It turns out to be much easier to use the most convenient of the many equivalent definition of amenability to do this. But before moving to these considerations, it's nice to wander a bit [3].

\subsection{Definition}

A group is called elementarily amenable (short notation: EA) if it is obtained by (many) applications of the closure properties 1)-4) starting from the following class of groups: finite groups and Z. F Day asked whether "amenable" = EA ("Day's conjecture"). Here are two important facts about EA groups [3] [5].

\section{Assumption}

Since $H$ denotes a Lie group, the ideas here are important even in the special case where $H$ is discrete [5].

\subsection{Definition [4]}

A group $\Gamma$ is amenable is there exists a state $\mu$ on $l^{\infty}(\Gamma)$ which is invariant 
under the left translation action: for all $s \in \Gamma$ and $f \in l^{\infty}(\Gamma)$, $\mu(s: f)=\mu(f)$.

Hence we can construct further examples from finite and abelian groups.

\subsection{Example}

Suppose $\Gamma$ is finitely generated by $S=\left\{s_{1}, \cdots, s_{d}\right\}$. One can then consider the Cayley graph of $\Gamma$ where vertices are group elements and edges connecting two group elements imply they differ by one of the generators in $S$. We place a metric on this graph by letting $d(s, t)$ by counting the "word length" of $s^{-1} t$. A property of interest is how $|B(e, r)|$, the ball centered at the identity element of radius $r$, varies with $r$, that is, the growth rate of the group [5].

It turns out that groups with sub exponential growth are always amenable.

\subsection{Example}

$\Gamma=\mathbb{F}_{2}$ is non-amenable: let $a, b \in \mathbb{F}_{2}$ be the two generators then let $A^{+}$is the set of all words starting with $a . A^{-1}$ is the set of all words starting with $a^{-1}$, and we define $B^{ \pm}$similarly. Lastly, we set $C=\left\{1, b, b^{2}, \cdots\right\}$. We note that we can decompose $\mathbb{F}_{2}$ in the three following ways:

$$
\begin{aligned}
\mathbb{F}_{2} & =A^{+} \sqcup A^{-1} \sqcup\left(B^{+} / C\right) \sqcup\left(B^{-} \cup C\right) \\
& =A^{+} \sqcup a A^{-1} \\
& =b^{-1}\left(B^{+} / C\right) \sqcup\left(B^{+} \cup C\right):
\end{aligned}
$$

If we had a state $\mu$ on $l^{\infty}(\Gamma)$ which was invariant under left translation then we would obtain:

$$
\begin{aligned}
1 & =\mu(1)=\mu\left(\chi A^{+}+\chi A^{-}+\chi B^{+} / C+\chi B^{-} \sqcup C\right) \\
& =\mu\left(\chi A^{+}\right)+\mu\left(\chi A^{-}\right)+\mu\left(\chi B^{+} / C\right)+\mu\left(\chi B^{-} / C\right) \\
& =\mu\left(\chi A^{+}\right)+a \cdot \chi A^{-}+\mu\left(b^{-1} \cdot \chi B^{+} / C\right)+\mu\left(\chi B^{-} \sqcup C\right) \\
& =\mu\left(\chi A^{+}\right)+a \chi A^{-}+\mu\left(\chi b^{-1} B^{+} / C\right)+\chi B^{-} \sqcup C \\
& =\mu(1)+\mu(1)=2 \mu(1),
\end{aligned}
$$

a contradiction [3] [5].

Our goal is to prove the following theorem:

\subsection{Theorem [5]}

For $\Gamma+$ a discrete group, the following are equivalent:

1) $\Gamma$ is amenable;

2) $\Gamma$ has an approximate invariant mean;

3) $\Gamma$ satisfies the F $\phi$ lner condition;

4) The trivial representation $\tau_{0}$ is weakly contained in the regular representation $\lambda$ (i.e., there exist unit vectors $\left.\xi_{i} \in l^{2}(\Gamma)\right)$ such that $\left\|\lambda_{s}\left(\xi_{i}\right)-\xi_{i}\right\|_{2} \rightarrow 0$ for all $s \in \Gamma)$,

5) There exists a net $(\varphi)$ of finitely supported positive definite functions on $\Gamma$, 
with $\varphi_{i}(e)=1$ for each $i$, such that $\varphi_{i} \rightarrow 1$ point wise;

6) $C^{*}(\Gamma)=C_{\lambda}^{*}(\Gamma)$;

7) $C_{\lambda}^{*}(\Gamma)$ has a character (i.e., one-dimensional representation);

8) for any finite subset $E \subset \Gamma$, we have

$$
\left\|\frac{1}{E} \sum_{s \in E} \lambda_{s}\right\|=1 .
$$

The main obstacle to proving this theorem is that we don't understand what most of it is saying. Consequently we'll parse the theorem as we go (rather than drowning the reader in definitions). The plan is to prove the cycle (1 22344567 ) and then $(48)$. We shall additionally prove $(4) \Rightarrow(6)$ in case the reader finds condition (5) distasteful [4].

\subsection{Definition [4]}

For a discrete group $\Gamma$, let $\operatorname{Prob}(\Gamma)$ be the space of all probability measures on $\Gamma$ :

$$
\operatorname{Prob}(\Gamma):=\left\{\mu \in t^{1}(\Gamma): \mu \geq 0 \text { and } \sum_{t \in \Gamma} \mu(t)=1\right\} .
$$

Then we say $\Gamma$ has an approximate invariant mean if for any finite subset $E \subset \Gamma$ and $\varepsilon>0$, there exists $\mu \in \operatorname{Prob}(\Gamma)$ such that

$$
\max _{s \in E}\|s \cdot \mu-\mu\|_{1}<\varepsilon
$$

Proof of $(1) \Rightarrow(2)$. Let $\mu$ be an invariant mean on $l^{\infty}(\Gamma)$. We claim there is a net $\left(\mu_{i}\right) \subset \operatorname{Prob}(\Gamma)$ which converges to $\mu$ weak $^{*}$ as elements of $l^{\infty}(\Gamma)^{*}$. Suppose not, then $\mu \notin \overline{\operatorname{Prob}(\Gamma)}{ }^{\omega^{*}}$ and since $\operatorname{Prob}(\Gamma)$ is convex the Hahn-Banach separation theorem implies there is some $f \in l^{\infty}(\Gamma)$ and $t<s \in \mathbb{R}$ such that

$$
\operatorname{Re}[v(f)]<t<s<\operatorname{Re}[\mu(f)],
$$

for all $v \in \operatorname{Prob}(\Gamma)$. Upon replacing $f$ with $\frac{f^{+}+\bar{f}}{2}$ we obtain $v(f)<t<s<\mu(f)$. Then replacing $f$ with $f+\|f\|_{\infty}$ ensures that $f \geq 0$. Consequently $\sup \{v(f): v \in \operatorname{Prob}(\Gamma)\}=\|f\|_{\infty}$ and yet

$$
\|f\|_{\infty}=\sup \{v(f): v \in \operatorname{Prob}(\Gamma)\} \leq t<s<\mu(f) \leq\|f\|_{\infty},
$$

a contradiction.

Hence we can find a net $\left(\mu_{i}\right)$ in $\operatorname{Prob}(\Gamma)$ which converges to $\mu$ in the weak topology. Thus for each $s \in \Gamma$ and $f \in l^{\infty}(\Gamma)$ we know $s \cdot \mu_{i}(f)-\mu_{i}(f) \rightarrow s \cdot \mu(f)-\mu(f)=\mu(f)-\mu(f)=0$. But since the $\mu_{i} \in l^{1}(\Gamma)$, this is equivalent to saying they converge weakly to zero in $l^{1}(\Gamma)$. Thus for any finite $E \subset \Gamma$, the weak closure of the convex subset $\oplus_{s \in E}\{s \cdot \mu-\mu: \mu \in \operatorname{Prob}(\Gamma)\}$ contains 0 . As a convex set, the weak and norm closures coincide by the Hahn-Banach theorem. Hence given $\varepsilon>0$ we can find $v \in \operatorname{Prob}(\Gamma)$ such that 


$$
\sum_{s \in E}\|s \cdot v-v-0\|_{1}<\varepsilon .
$$

Hence we have an approximate invariant mean [3].

\subsection{Definition [5]}

We say $\Gamma$ satisfies the F $\phi$ lner condition if for any finite $E \subset \Gamma$ and $\varepsilon>0$, there exists a finite subset $E \subset \Gamma$ such that

$$
\max _{s \in E} \frac{|s F \Delta F|}{|F|}<\varepsilon \text {. }
$$

That is, the action of $E$ does not move $F$ around "too much." Furthermore, a sequence of finite sets $F_{n} \in \Gamma$ such that

$$
\frac{\left|s F_{n} \Delta F_{n}\right|}{\left|F_{n}\right|} \rightarrow 0
$$

is called a $F \phi l n e r$ sequence.

Proof of (2) $\Rightarrow$ (3). Fix a finite subset $E \subset \Gamma$ and $\varepsilon>0$. Since we have an approximate invariant mean we can find $\mu \in \operatorname{Prob}(\Gamma)$ such that

$$
\sum_{s \in E}\|s \cdot \mu-\mu\|_{1}<\varepsilon \text {. }
$$

Given a positive function $f \in l^{1}(\Gamma)$ and $r \geq 0$, we define a set $F(f ; r):=\{t \in \Gamma: f(t)>r\}$. Now, note that for a pair of positive functions $f, h \in l^{1}(\Gamma)$ and $t \in \Gamma,\left|\chi_{F(f, r)}(t)-\chi_{F(h ; r)}(t)\right|=1$ if and only if $r$ lies between the numbers $f(t)$ and $h(t)$. Furthermore, if $f$ and $h$ are bounded above by 1 then it follows that

$$
|f(t)-h(t)|=\int_{0}^{1}\left|\chi_{F(f, r)}(t)-\chi_{F(h ; r)}(t)\right| \mathrm{d} r
$$

We apply this to $\mu$ and $s \cdot \mu$ to get

$$
\begin{aligned}
\|s \cdot \mu-\mu\|_{1} & =\sum_{t \in \Gamma}|s \cdot \mu(t)-\mu(t)|=\sum_{t \in \Gamma} \int_{0}^{1}\left|\chi_{F(s \cdot \mu, r)}(t)-\chi_{F(\mu, r)}(t)\right| \mathrm{d} r \\
& =\int_{0}^{1} \sum_{t \in \Gamma}\left|\chi_{F(s \cdot \mu, r)}(t)-\chi_{F(\mu, r)}(t)\right| \mathrm{d} r=\int_{0}^{1}|s F(\mu, r) \Delta F(\mu, r)| \mathrm{d} r
\end{aligned}
$$

Also, we have

$$
\begin{aligned}
\int_{0}^{1}|F(\mu, r)| \mathrm{d} r & =\int_{0}^{1} \sum_{t \in \Gamma}\left|\chi_{F(\mu, r)}(t)\right| \mathrm{d} r=\sum_{t \in \Gamma} \int_{0}^{1}\left|\chi_{F(\mu, r)}(t)\right| \mathrm{d} r \\
& =\sum_{t \in \Gamma} \int_{0}^{\mu(t)} 1 \mathrm{~d} r=\sum_{t \in \Gamma} \mu(t)=1
\end{aligned}
$$

Thus

$\epsilon \int_{0}^{1}|F(\mu, r)| \mathrm{d} r=\epsilon>\sum_{s \in E}\|s \cdot \mu(t)-\mu(t)\|_{1}=\int_{0}^{1} \sum_{s \in E}|s F(\mu, r) \Delta F(\mu, r)| \mathrm{d} r$

So for some $\mathrm{r}$ we must have

$$
\sum_{s \in E}|s F(\mu, r) \Delta F(\mu, r)|<\epsilon|F(\mu, r)| .
$$

Letting $F:=F(\mu, r)$ we are done. 
For the next implication we will ignore the first version of the statement and instead focus on the later, equivalent condition. We only need to understand the left regular representation. This is a homomorphism $\lambda: \Gamma \rightarrow u\left(I^{2}(\Gamma)\right)$ where the image of $s \in \Gamma$ is denoted $\lambda_{s}$ and for $f \in l^{2}(\Gamma)\left(\lambda_{s} f\right)(t)=f\left(s^{-1} t\right)$.

Proof of $(3) \Rightarrow(4)$. From the F $\phi$ lner condition build a F $\phi$ lner sequence $\left(F_{i}\right)$ (by letting $\left.\epsilon=1, \frac{1}{2}, \frac{1}{3}, \cdots\right)$ set $\xi_{i}:=\left|F_{i}\right|^{-1 / 2} \chi F_{i}$. Then $\xi \in l^{2}(\Gamma)$ are unit vectors and

$$
\begin{aligned}
& \| \lambda_{s}\left(\xi_{i}\right)-\left.\xi_{i}\right|_{2} ^{2}=\sum_{t \in \Gamma}\left|\lambda_{s}\left(\xi_{i}\right)(t)-\xi_{i}(t)\right|^{2} \\
& =\sum_{t \in \Gamma}\left|\frac{1}{\left|F_{i}\right|^{1 / 2}} \chi F_{i}\left(s^{-1} t\right)-\frac{1}{\left|F_{i}\right|^{1 / 2}} \chi F_{i}(t)\right|^{2} \\
& =\frac{1}{\left|F_{i}\right|} \sum_{t \in \Gamma}\left|\chi_{s F_{i}}(t)-\chi_{F_{i}}(t)\right|^{2}=\frac{\left|s F_{i} \Delta F_{i}\right|}{\left|F_{i}\right|} \rightarrow 0 .
\end{aligned}
$$

\subsection{Definition [6]}

A function $\varphi: \Gamma \rightarrow \mathbb{C}$ is called positive definite if the matrix

$$
\left[\varphi\left(s^{-1} t\right)\right]_{s, t \in F} \in M_{|F| \times|F|}(\mathbb{C}),
$$

is positive for every finite set $F \subset \Gamma$.

Proof $(4) \Rightarrow(5)$. For a unit vector $\xi \in l^{2}(\Gamma)$, define $\varphi(s):=\left\langle\lambda_{s} \xi, \xi\right\rangle_{l^{2}(\Gamma)}$. Then we claim that $\varphi$ is positive definite. Indeed,

$$
\left[\varphi\left(s^{-1} t\right)\right]=\left[\left\langle\lambda_{s^{-1} t} \xi, \xi\right\rangle\right]=\left[\left\langle\lambda_{s}^{*} \lambda_{t} \xi, \xi\right\rangle\right]=\left[\left\langle\lambda_{t} \xi, \lambda_{s} \xi\right\rangle\right],
$$

Let $n=|F|, F=\left\{t_{1}, \cdots, t_{n}\right\}$, and fix $v=\left(z_{1}, \cdots, z_{n}\right) \in \mathbb{C}^{n}$. It suffices to show:

$$
\begin{aligned}
{\left[\left\langle\lambda_{t} \xi, \lambda_{s} \xi\right\rangle\right] v } & =\left[\begin{array}{ccc}
\left\langle\lambda_{t_{1}} \xi, \lambda_{t_{1}} \xi\right\rangle & \cdots & \left\langle\lambda_{t_{n}} \xi, \lambda_{t_{1}} \xi\right\rangle \\
\vdots & \ddots & \vdots \\
\left\langle\lambda_{t_{1}} \xi, \lambda_{t_{n}} \xi\right\rangle & \cdots & \left\langle\lambda_{t_{n}} \xi, \lambda_{t_{n}} \xi\right\rangle
\end{array}\right]\left(\begin{array}{c}
z_{1} \\
\vdots \\
z_{n}
\end{array}\right) \\
& =\left(\begin{array}{c}
\left\langle\lambda_{t_{1}} \xi, \lambda_{t_{1}} \xi\right\rangle z_{1}+\cdots+\left\langle\lambda_{t_{n}} \xi, \lambda_{t_{1}} \xi\right\rangle z_{n} \\
\vdots \\
\left\langle z_{1} \lambda_{t_{1}} \xi, \lambda_{t_{n}} \xi\right\rangle+\cdots+\left\langle\lambda_{t_{n}} \xi, \lambda_{t_{1}} \xi\right\rangle z_{n}
\end{array}\right) \\
& =\left(\begin{array}{c}
\left\langle\lambda_{t_{1}} \xi, \lambda_{t_{1}} \xi\right\rangle z_{1}+\cdots+\left\langle z_{n} \lambda_{t_{n}} \xi, \lambda_{t_{1}} \xi\right\rangle \\
\vdots \\
\left\langle z_{1} \lambda_{t_{1}} \xi, \lambda_{t_{1}} \xi\right\rangle+\cdots+\left\langle z_{n} \lambda_{t_{n}} \xi, \lambda_{t_{1}} \xi\right\rangle
\end{array}\right) \\
& =\left(\begin{array}{c}
\sum_{i=1}^{n}\left\langle z_{i} \lambda_{t_{i}} \xi, \lambda_{t_{1}} \xi\right\rangle \\
\vdots \\
\sum_{i=1}^{n}\left\langle z_{i} \lambda_{t_{i}} \xi, \lambda_{t_{1}} \xi\right\rangle
\end{array}\right)
\end{aligned}
$$

and so 


$$
\begin{aligned}
\left\langle\left[\lambda_{t} \xi, \lambda_{s} \xi\right] v, v\right\rangle & =\left(\begin{array}{c}
\sum_{i=1}^{n}\left\langle z_{i} \lambda_{t_{i}} \xi, \lambda_{t_{1}} \xi\right\rangle \\
\vdots \\
\sum_{i=1}^{n}\left\langle z_{i} \lambda_{t_{i}} \xi, \lambda_{t_{1}} \xi\right\rangle
\end{array}\right) \cdot\left(\begin{array}{c}
z_{1} \\
\vdots \\
z_{n}
\end{array}\right) \\
& =\sum_{j=1}^{n} \sum_{i=1}^{n}\left\langle z_{i} \lambda_{t_{i}} \xi, \lambda_{t_{j}} \xi\right\rangle \bar{z}_{j}=\sum_{j=1}^{n} \sum_{i=1}^{n}\left\langle z_{i} \lambda_{t_{i}} \xi, \lambda_{t_{j}} \xi\right\rangle \\
& =\left\langle\sum_{i=1}^{n} z_{i} \lambda_{t_{i}} \xi \sum_{j=1}^{n} z_{i} \lambda_{t_{j}} \xi\right\rangle=\left\|\sum_{i=1}^{n} z_{i} \lambda_{t_{i}} \xi\right\|^{2} \geq 0
\end{aligned}
$$

Hence $\varphi$ is positive definite. So letting $\left(\xi_{i}\right)$ be the unit vectors from condition (4) and setting $\varphi_{i}(s):=\left\langle\lambda_{s} \xi_{i}, \xi_{i}\right\rangle$ we know $\varphi_{i}(e)=1$ and from our above work that these functions are positive definite. From condition (4) we also know that they converge pointwise to 1 . In order to make them finitely supported we need merely replace the $\xi_{i}$ with finitely supported elements.

Starting with a discrete group $\Gamma$ we can consider the group algebra $\mathbb{C}[\Gamma]=\left\{\sum_{i=1}^{n} \alpha_{i} \cdot t_{i}: n \in \mathbb{N}, \alpha_{t} \in \mathbb{C}, t_{i} \in \Gamma\right\}$ with addition and multiplication defined in the obvious ways and an involution defined by

$$
\left(\sum_{i=1}^{n} \alpha_{i} \cdot t_{i}\right)^{*}=\sum_{i=1}^{n} \bar{\alpha}_{i} \cdot t_{i}^{-1}
$$

We want to extend this into a $C$-algebra, but there are multiple norms we use. On the one hand we can extend the left regular representation $\lambda$ to a *-representation of $\mathbb{C}[\Gamma]$ on $l^{2}(\Gamma)$, still denoted by $\lambda$, by

$$
\lambda\left(\sum_{i=1}^{n} \alpha_{i} \cdot t_{i}\right)^{*}=\sum_{i=1}^{n} \alpha_{i} \cdot \lambda_{t_{i}} B \in\left(l^{2}(\Gamma)\right) .
$$

The reduced $C$-algebra is then what we obtain by taking the closure of $\lambda(\mathbb{C}[\Gamma])$ with respect to $\|\cdot\|_{\in\left(I^{2}(\Gamma)\right)}$, we denote it by $C_{\lambda}^{*}(\Gamma)$. On the other hand, the left regular representation $\lambda: \Gamma \rightarrow u\left(I^{2}(\Gamma)\right)$ is merely one representation of our group. Hence we can consider the norm

$$
\|x\|_{u}=\sup \left\{\|\pi(x)\|_{B(H)}: \pi: \Gamma \rightarrow u(\mathcal{H}) \text { is a } * \text {-representation }\right\} .
$$

This easily satisfies the $C$-identity. The full (or universal) $C$-algebra of $\Gamma$ is the closure of $\mathbb{C}[\Gamma]$ with respect to $\|\cdot\|_{u}$ and is denoted $C^{*}(\Gamma)$ [2].

Thus assuming (5) we'll need to show that these two $C$-algebras coincide. But we first note that since $\|\lambda(x)\| \leq\|x\|_{u}$ for $x \in \mathbb{C}[\Gamma], \lambda$ extends to $C^{*}(\Gamma)$. It is clear that this is onto $C_{\lambda}^{*}(\Gamma)$ [7].

\subsection{Definition [6]}

Let $\varphi: \Gamma \rightarrow \mathbb{C}$ be a function. The associated multiplier $m_{\varphi}: \mathbb{C}[\Gamma] \rightarrow \mathbb{C}[\Gamma]$ is defined by

$$
m_{\varphi}\left(\sum_{t \in \Gamma} \alpha_{t} \cdot t\right):=\sum_{t \in \Gamma} \varphi(t) \alpha_{t} \cdot t .
$$

We also define $\tilde{m}_{\varphi}: \lambda(\mathbb{C}[\Gamma]) \rightarrow \lambda(\mathbb{C}[\Gamma])$ by

$$
\tilde{m}_{\varphi}\left(\lambda\left(\sum_{t \in \Gamma} \alpha_{t} \cdot t\right)\right)=\tilde{m}_{\varphi}\left(\sum_{t \in \Gamma} \alpha_{t} \lambda_{t}\right)=\sum_{t \in \Gamma} \varphi(t) \alpha_{t} \lambda_{t} .
$$




\subsection{Lemma}

Suppose $\varphi$ is finitely supported, positive definite, and $\varphi(e)=1$. Then $m_{\varphi}$ extends to a continuous map on $C^{*}(\Gamma)$ and $\tilde{m}_{\varphi}$ extends to a continuous map on $C_{\lambda}^{*}(\Gamma)$. both with norm one.

Proof. First consider the case when $\varphi=\delta_{e}$ (i.e. $\varphi(t)=1$ if $t=e$ and $\varphi(t)=0$ otherwise). Let $\tau(x)=\left\langle\lambda(x) \delta_{e}, \delta_{e}\right\rangle_{1^{2}(\Gamma)}$ for $x \in C^{*}(\Gamma)$, then $\tau$ is a tracial state. For $x \in \mathbb{C}[\Gamma]$ we compute:

$$
\left\|m_{\varphi}(x)\right\|_{u}=\|\tau(x) \cdot e\|_{u}=|\tau(x)|\|e\|_{u}=|\tau(x)| \leq\|x\|_{u} .
$$

Hence we can extend $m_{\varphi}$ to $C^{*}(\Gamma)$ with norm one.

Let $\tilde{\tau}(T)=\left\langle T \delta_{e}, \delta_{e}\right\rangle_{I^{2}(\Gamma)}$ for $T \in B\left(C^{*}(\Gamma)\right)$, then $\tilde{\tau}$ is a tracial state. For $x \in \mathbb{C}[\Gamma]$ we compute:

$$
\left\|\tilde{m}_{\varphi}(\lambda(x))\right\|=\left\|\tilde{\tau}(\lambda(x)) \lambda_{e}\right\|=|\tilde{\tau}(\lambda(x))|\left\|\lambda_{e}\right\|=|\tilde{\tau}(\lambda(x))| \leq\|\lambda x\| .
$$

so that $\tilde{m}_{\varphi}$ extends to $C_{\lambda}^{*}(\Gamma)$ with norm one.

Next consider the case $\varphi=\delta_{t}$ for $t \in \Gamma$. Since

$$
\begin{aligned}
\left\|m_{\varphi}(x)\right\|_{u} & =\left\|\left\langle\lambda(x) \delta_{e}, \delta_{t}\right\rangle \cdot t\right\|_{u}=\left\|\left\langle\lambda(x) \delta_{e}, \lambda \delta_{t}\right\rangle \cdot t\right\|_{u} \\
& =\left\|\left\langle\lambda_{t^{-1}} \lambda(x) \delta_{e}, \delta_{e}\right\rangle \cdot t\right\|_{u}=\left\|\tau\left(t^{-1} x\right) \cdot t\right\|_{u} \\
& =\mid \tau\left(t^{-1} x\right)\|t\| t\left\|_{u} \leq\right\| t^{-1} x\left\|_{u} \leq\right\| x \|_{u},
\end{aligned}
$$

we see that $m_{\varphi}$ again extends to $C^{*}(\Gamma)$ with norm one. A similar computation for $\tilde{m}_{\varphi}$ involving $\tilde{\tau}$ yields an extension in the reduced $C$-algebra case as well.

Thus for a finitely supported $\varphi$ we can write $\varphi=\sum_{t \in \Gamma} \varphi(t) \delta_{t}$ and so $m_{\varphi}=\sum_{t \in \Gamma} \varphi(t) m_{\delta_{t}}$. Extending each of the finitely many $m_{\delta_{t}}$ yields an extension for $m_{\varphi}$. But since $\varphi$ is positive definite, $m_{\varphi}$ is positive and hence attains its norm at the identity: $\left\|m_{\varphi}\right\|=\left\|m_{\varphi}(e)\right\|=|\varphi(e)|=1$. A similar argument applies in the reduced $C$-algebra case.

Proof of $(5) \Rightarrow(6)$. By our previous comments, we know $\lambda: C^{*}(\Gamma) \rightarrow C_{\lambda}^{*}(\Gamma)$ is onto and hence it remains to show $\lambda$ is injective.

Let $\left(\varphi_{i}\right)$ be the net in condition (5). By the above lemma, we can define multipliers $m_{\varphi_{i}}$ and $\tilde{m}_{\varphi_{i}}$ on $C^{*}(\Gamma)$ and $C_{\lambda}^{*}(\Gamma)$ respectively, each with norm one. We note that $\lambda \circ m_{\varphi_{i}}=\tilde{m}_{\varphi_{i}} \circ \lambda$ on $C^{*}(\Gamma)$ since both functions are continuous and agree on the dense subspace $\mathbb{C}[\Gamma]$. Now, since $\varphi_{i} \rightarrow 1$ pointwise on $\Gamma, m_{\varphi_{i}}(x) \rightarrow x$ for $x \in \mathbb{C}[\Gamma]$. Since the norms of the $m_{\varphi_{i}}$ are uniformly bounded by one and $\mathbb{C}[\Gamma]$ is dense in $C^{*}(\Gamma)$, this limit holds for $x \in C^{*}(\Gamma)$ as well.

Now, suppose $x \in C^{*}(\Gamma)$ and $\lambda(x)=0$. Then

$$
\lambda\left(m_{\varphi_{i}}(x)\right)=\tilde{m}_{\varphi_{i}}(\lambda(x))=0,
$$

for every $i$. But since $\varphi_{i}$ is finitely supported we know $m_{\varphi_{i}}(x) \in \mathbb{C}[\Gamma]$ and hence $\lambda\left(m_{\varphi_{i}}(x)\right)=0$ implies $m_{\varphi_{i}}(x)=0$. Hence $x=\lim _{i} m_{\varphi_{i}}(x)=0$ and so $\lambda$ is injective. 
Proof of $(6) \Rightarrow(7) . C^{*}(\Gamma)$ always has a one-dimensional representation since the trivial representation $\mathbb{C}[\Gamma] \ni \sum_{t} \alpha_{t} \cdot t \mapsto \sum_{t} \alpha_{t} \in \mathbb{C}$ is always subordinate to $\|\cdot\|_{u}$. Hence $C_{\lambda}^{*}(\Gamma)=C^{*}(\Gamma)$ has a character.

We require a lemma:

\section{Definition [6]}

Suppose $H$ acts continuously on a locally convex topological vector space $\mathcal{V}$. Every $H$-invariant, compact, convex subset of $\mathcal{V}$ is called a compact, convex $H$-space.

\subsection{Definition}

$H$ is amenable if and only if $H$ has a fixed point in every nonempty, compact, convex $H$-space. This is just one of many different equivalent definetions of amenability. The equivalence of these diverse definitions is a testament to the fact that this notion is very fundamental [6].

\subsection{Remarks}

1) All locally convex topological vector spaces are assumed to be Hausdorff.

2) In most applications, the locally convex space $V$ is the dual of a separable Banach space, with the weak topology [6].

In this situation, every compact, convex subset $C$ is second countable, and is therefore metrizability. With these thoughts in mind, we feel free to assume metrizability when it eliminates technical difficulties in our proofs. In fact, we could restrict to these spaces in the definition of amenability, because it turns out that this modified definition results in exactly the same class of groups.

3) The choice of the term "amenable" seems to have been motivated by two considerations:

a) The word "amenable" can be pronounced "a-MEAN-able," and we will see that a group is amenable if and only if it admits certain types of means.

b) One definition of "amenable" from the Oxford American Dictionary is capable of being acted on a particular way. "In other words, in colloquial English, something is lamenable" if it is easy to work with. Classical analysis has averaging theorems and other techniques that were developed for the study of functions on the group Rn. Many of these methods can be generalized to all amenable groups, so amenable groups are easy to work with [6].

\section{Examples of Amenable Groups}

1) Abelian groups are amenable.

2) Compact groups are amenable.

3) Solvable groups are amenable, because the class of amenable groups is closed under extensions.

4) Closed subgroups of amenable groups are amenable.

On the other hand, however, it is important to realize that not all groups are 
amenable. In particular, we will see that:

a) nonabelian free groups are not amenable, and

b) $S L(2 ; R)$ is not amenable.

We begin by showing that $Z$ is amenable [8].

\subsection{Proposition}

Cyclic groups are amenable.

Proof. Assume $H=\langle T\rangle$ is cyclic. Given a nonempty, compact, convex $H$-space $C$, choose some $c_{0} \in C$. For $n \in \mathbb{N}$, let

$$
c_{n}=\frac{1}{n+1} \sum_{k=0}^{n} T^{k}(c)
$$

Since $C$ is compact, the sequence $\left\{c_{n}\right\}$ must have an accumulation point.

$c \in C$. It is not difficult to see that $c$ is fixed by. Since $T$ generates $H$, this means that $c$ is a fixed point for $H$.

\subsection{Corollary}

(Kakutani-Markov Fixed Point Theorem). Every abelian group is amenable.

Proof. Let us assume $H=\langle g, h\rangle$ is a 2-generated abelian group. [5] If $C$ is any nonempty, compact, convex $H$-space, then Proposition 2.1 implies that the set $C^{g}$ of fixed points of $g$ is nonempty. It is easy to see that $C^{g}$ is compact and convex, and, because $H$ is abelian, that $C^{g}$ is invariant under $h$. Hence, $C^{g}$ is a nonempty, compact, convex $\langle h\rangle$-space. Therefore, Pro-position2.1 implies that $h$ has a fixed point $c$ in $C^{g}$. Now $c$ is fixed by $g$, and $c$ is fixed by $h$, so $c$ is fixed by $\langle g, h\rangle=H \quad[8]$.

Compact groups are also easy to work with.

\subsection{Proposition}

Compact groups are amenable.

Proof. Assume $H$ is compact, and let $\mu$ be a Haar measure on $H$. Given a nonempty, compact, convex $H$-space $C$, choose some $c_{0} \in C$. Since $\mu$ is a probability measure, we may let probability measure, we may let

$$
c=\int_{H} h\left(c_{0}\right) \mathrm{d} \mu(h) \in C .
$$

The $H$-invariance of $\mu$ implies that $c$ is a fixed point for $H$ ).

It is easy to show that amenable extensions of amenable groups are amenable here, Let $1 \rightarrow N \rightarrow G \rightarrow Q \rightarrow 1$ be an extension of (discrete) groups, where $N$ and $Q$ are amenable [9]

\section{Invariant Probability Measures}

\subsection{Definitions}

Let $X$ be a complete metric space.

1) A measure $\mu$ on $X$ is a probability measure if $\mu(X)=1$. A probability 
measure is a measure with total measure one, $\mu(X)=1$. A probability space is a measure space with a probability measure. For measure spaces that are also topological spaces various compatibility conditions can be placed for the measure and the topology.

2) $\operatorname{Prob}(X)$ denotes the space of all probability measures on $X$. Any measure on $X$ is also a measure on the one-point compact fication $X^{7}$ of $X$, so, if $X$ is locally compact, then the Riesz Representation Theorem tells us that every finite measure on $X$ can be thought of as a linear functional on the Banach space $C\left(X^{+}\right)$of continuous functions on $X^{*}$. This implies that $\operatorname{Prob}(X)$ is a subset of the closed unit ball in the dual space $C\left(X^{+}\right)^{*}$, and therefore has a weak topology. If $X$ is compact, then the Banach-Alaoglu Theorem (B7.4) tells us that $\operatorname{Prob}(X)$ is compact [9].

\subsection{Example}

If a group $H$ acts continuously on a compact, metrizable space $X$, then $\operatorname{Prob}(X)$ is a compact, convex $H$-space [9].

\subsection{Remark}

Recall that a compact, Hausdorff space is metrizable if and only if it is second countable, so requiring a compact, separable, Hausdorff space to be metrizable is not a strong restriction [9].

\subsection{Proposition $(1 \Leftrightarrow 3)$}

$H$ is amenable if and only if for every continuous action of $H$ on a compact, metrizable space $X$, there is an $H$-invariant probability measure $\mu$ on $X$.

Proof. $(\Longrightarrow)$ If $H$ acts on $X$, and $X$ is compact, then $\operatorname{Prob}(X)$ is a nonempty, compact, convex $H$-space. So $H$ has a fixed point in $\operatorname{Prob}(X)$; this fixed point is the desired $H$-invariant measure. $(\Leftarrow)$ Suppose $C$ is a nonempty, compact, convex $H$-space. By replacing $C$ with the closure of the convex hull of a single $H$-orbit, we may assume $C$ is separable; then $C$ is metrizable. Since $H$ is amenable, this implies there is an $H$-invariant probability measure $\mu$ on $C$. Since $C$ is convex and compact, the center of mass

$$
p=\int_{c} c \mathrm{~d} \mu(c)
$$

belongs to $C$. Since $\mu$ is $H$-invariant, a simple calculation shows that $p$ is $H$-invariant [9].

\section{Invariant Means}

\subsection{Definition}

Suppose $\mathcal{V}$ is some linear subspace of $\mathcal{L}^{\infty}(H)$, and assume $\mathcal{V}$ contains the constant function $1_{H}$ that takes the value 1 at every point of $H$. A mean on $\mathcal{V}$ is a linear functional $\lambda$ on $\mathcal{V}$, such that ${ }^{*} \lambda\left(1_{H}\right)=1$, and ${ }^{*} \lambda$ is positive, i.e., $\lambda(f) \geq 0$ whenever $f \geq 0$. 


\subsection{Remark}

Any mean is a continuous linear functional; indeed, $\|\lambda\|=1$.

It is easy to construct means.

\subsection{Example}

If $\phi$ is any unit vector in $\mathcal{L}^{1}(H)$, and $\mu$ is the left Haar measure on $H$, then defining

$$
\lambda(f)=\int_{H} f(\phi) \mathrm{d} \mu
$$

produces a mean (on any subspace of $\mathcal{L}^{\infty}(H)$ that contains $1_{H}$ ). Means constructed in this way are (weakly) dense in the set of all means. Compact groups are the only ones with invariant probability measures, but invariant means exist more generally [10].

\section{Acknowledgements}

We would like to thank the all library staff for the references that we use. We are also grateful to the professors who provided us with useful information in the author of this paper.

\section{Conflicts of Interest}

The authors declare no conflicts of interest regarding the publication of this paper.

\section{References}

[1] Wehrfritz, B.A.F. (1969) In_nite Linear Groups. Springer, Berlin.

[2] Luxemburg, W.A.J. (1969) Applications of Model Theory to Algebra, Analysis, and Probability. Holt, Rinehart and Winston, New York.

[3] Neumnn, H. (1967) Varieties of Groups, Springer-Verlag, New York. https://doi.org/10.1007/978-3-642-88599-0

[4] Arveson, W. (1976) An Invitation to $C^{*}$-Algebras. Springer, New York.

[5] Bekka, B., de la Harpe, P. and Valette, A. (2008) Kazhdan's Property (T). Cambridge U. Press, Cambridge.

[6] Dy, M.M. (1957) Amenable Semigroups. Illinois Journal of Mathematics, 1, 509-544. https://doi.org/10.1215/ijm/1255380675

[7] Jean-Marie Normand Service de Physique Theorique, CEA/DSM/SPhT CNRS/ SPM/URA 2306 CEA/Saclay, F-91191 Gif-sur-Yvette Cedex, France.

[8] Greenleaf, F.P. (1969) Invariant Means on Topological Groups. Van Nostrand, New York.

[9] Fclnr, E. (1955) On Groups with Full Banach Mean Values. Mathematica Scandinavica, 3, 243-254. https://doi.org/10.7146/math.scand.a-10442

[10] Brown, N.P. and Ozawa, N. (2008) $C^{*}$-Algebras and Finite-Dimensional Approximations. Graduate Studies in Mathematics, 88.

https://doi.org/10.1090/gsm/088 